\title{
Criminologie
}

\section{Les instruments d'évaluation du risque de comportements violents : mise en perspective critique}

\section{Gilles Côté}

Volume 34, numéro 1, printemps 2001

La notion de risque dans la gestion pénale

URI : https://id.erudit.org/iderudit/004752ar

DOI : https://doi.org/10.7202/004752ar

Aller au sommaire du numéro

Éditeur(s)

Les Presses de l'Université de Montréal

ISSN

0316-0041 (imprimé)

1492-1367 (numérique)

Découvrir la revue

Citer cet article

Côté, G. (2001). Les instruments d'évaluation du risque de comportements violents : mise en perspective critique. Criminologie, 34(1), 31-45.

https://doi.org/10.7202/004752ar
Résumé de l'article

La capacité des cliniciens d'évaluer la dangerosité d'un individu a été sérieusement mise en doute depuis le début des années 1960. Devant cette conclusion appuyée par plusieurs études, des instruments ont été développés pour pallier le jugement clinique. Deux grandes tendances sont observées. L'une aborde le problème sous un angle actuariel, soit purement statistique. L'autre ajoute aux variables statiques des indices dynamiques liés à des aspects cliniques et au contexte futur dans lequel la personne est appelée à vivre. Ces deux tendances soulèvent des questions aux plans méthodologique, épistémologique et éthique. 


\title{
Les instruments d'évaluation du risque de comportements violents : mise en perspective critique
}

\author{
Gilles Côté \\ Directeur \\ Centre de recherche de l'Institut Philippe Pinel de Montréal \\ Professeur \\ Département de psychologie \\ Université du Québec à Trois-Rivières \\ gilles_cote@uqtr.uquebec.ca
}

RÉSUMÉ - La capacité des cliniciens d'évaluer la dangerosité d'un individu a été sérieusement mise en doute depuis le début des années 1960. Devant cette conclusion appuyée par plusieurs études, des instruments ont été développés pour pallier le jugement clinique. Deux grandes tendances sont observées. L'une aborde le problème sous un angle actuariel, soit purement statistique. L'autre ajoute aux variables statiques des indices dynamiques liés à des aspects cliniques et au contexte futur dans lequel la personne est appelée à vivre. Ces deux tendances soulèvent des questions aux plans méthodologique, épistémologique et éthique.

ABSTRACT - The ability of clinicians to assess the dangerousness of an individual has been seriously questioned since the early 1960s. In the face of this conclusion corroborated by various studies, a number of instruments have been developed to buttress clinical judgement. Two major trends have emerged in this regard. The first approaches the problem from an actuarial or purely statistical standpoint. The second complements static variables with dynamic indices related to clinical dimensions and to the future context in which the person will be called upon to live. These two tendencies raise certain methodological, epistemological and ethical questions. 
La mise en perspective critique des instruments d'évaluation du risque de comportements violents active parfois un certain nombre de réactions ; certains réagissent à ces instruments qui viennent questionner, voire porter ombrage, à une certaine pratique clinique. Les instruments actuariels ont été qualifiés de simplistes par certains (commentaire rapporté par Grann et al., 2000 : 99), de trop complexes par d'autres (commentaire rapporté par Quinsey et al., 1998 : 177), alors que quelques cliniciens y perçoivent une approche déshumanisante (commentaire rapporté par Heilbrun et al., 1999 : 405). Pour certains, ces démarches viennent questionner leur propre identité professionnelle en tant que clinicien (à partir des commentaires rapportés par Heilbrun et al., 1999 : 405).

\section{Développement des instruments d'évaluation du risque de comportements violents}

Les cliniciens engagés dans le champ des troubles mentaux sévères et persistants, tout comme ceux qui interviennent principalement dans le champ des troubles de la personnalité, sont régulièrement appelés à évaluer le risque de comportements violents dont pourraient se rendre responsables leurs clients. Les premières études qui ont cherché à estimer la capacité des cliniciens à prédire la «dangerosité » ont brossé un tableau assez pessimiste de la capacité des cliniciens à ce faire, pessimisme qui a prévalu au cours des années 60 à 80 . Plusieurs auteurs demeurent réservés sur la capacité des cliniciens à évaluer la dangerosité (Quinsey et al., 1998 ; Rabinowitz et Garelik-Wyler, 1999). Dans un ouvrage maintenant classique, Monahan (1981) estime que l'évaluation de la dangerosité établie par un clinicien est exacte une fois sur trois. Dans les faits, les cliniciens sont portés à surévaluer le nombre de patients qui présentent un danger, ce qu'a clairement démontré le suivi des cas dits Baxstrom (Steadman et Cocozza, 1974) et celui des cas dits Dixon (Thornberry et Jacoby, 1979). Suite à la décision de la Cour suprême des États-Unis dans le premier cas, et d'une cour fédérale dans le second cas, il fut reconnu que l'hospitalisation involontaire de ces derniers patients était anticonstitutionnelle. Dans le cas des sujets Baxstrom, 20 personnes récidivèrent sur les 98 sujets de l'échantillon, sujets jugés dangereux à l'origine. Généralement, il s'agissait de méfaits liés au vagabondage et à l'intoxication ; moins de $5 \%$ furent condamnés à nouveau pour un délit sérieux ("felony ») à un moment ou à un autre au 
TAB LEA U 1

Items constituant le Violence Risk Appraisal Guide (VRAG)

\begin{tabular}{|ll|}
\hline 1) & Psychopathie (PCL-R) \\
2) & Inadaptation scolaire à l'élémentaire \\
3) & Trouble de personnalité \\
4) & Âge au moment du délit \\
5) & Séparation de l'un ou l'autre de ses \\
& parents (exception de la mort) avant 16 ans \\
6) & Manquement antérieur à une libération sur parole \\
7) & Histoire de délits non violents \\
& $\begin{array}{l}\text { (selon échelle de Cormier-Lang) } \\
\text { 8) }\end{array}$ \\
N'a jamais été marié(e) \\
(ou équivalent) \\
Schizophrénie \\
Blessure la plus sérieuse infligée \\
à une victime (délit actuel) \\
Histoire d'abus d'alcool \\
12) & Victime du délit actuel est une femme \\
\hline
\end{tabular}

cours des quatre ans et demi qu'a duré le suivi. Dans le cas des sujets Dixon, 23,7 \% des 414 patients suivis récidivèrent ; toutefois, la récidive violente ne fut observée que chez $13,8 \%$ des patients.

Une seconde génération de recherches a pris naissance au début des années 1980, sous l'influence de John Monahan. Les pressions légales, notamment liées à l'hospitalisation non volontaire d'une personne atteinte d'un trouble mental, ont conduit à l'émergence d'instruments pour l'évaluation du "risque ». Il ne s'agit plus d'évaluer si le patient est dangereux ou non, mais d'estimer le risque de comportements violents pour un individu donné, dans un contexte donné, et selon un temps donné.

Divers instruments ont ainsi pris forme (Sex Offender Risk Appraisal Guide (SORAG) : Quinsey et al., 1998 ; Violence Risk Appraisal Guide (VRAG) : Quinsey et al., 1998 ; Psychopathy Checklist-Revised (Hare, 1991) ; HCR-20 : Assessing Risk for Violence, deuxième version : Webster et al. (1997) ; Sexual Violence Risk (SVR-20) : Boer et al., 1997 ; Spousal Assault Risk Assessment Guide (SARA) : Kropp et al., 1995 ; Estimate of Suicide Risk (ESR) : Polvi, 1997 ; grille actuarielle de McNiel et Binder (1994) pour prédire les comportements violents sur les unités psychiatriques ; etc. (voir entre autres les échelles actuarielles pour prédire les comportements sexuels délictuels auxquelles réferent Hanson et Thornston, 2000)). Dans 
TABLEA U 2

Items constituant la HCR-20

\begin{tabular}{|c|c|c|c|c|c|}
\hline \multicolumn{2}{|r|}{ Facteurs historiques } & \multicolumn{2}{|r|}{ Facteurs cliniques } & \multicolumn{2}{|r|}{ Gestion du risque } \\
\hline H1 & Violence antérieure & C1 & Introspection difficile & R1 & Plans irréalisables \\
\hline H2 & $\begin{array}{l}\text { Premier acte de } \\
\text { violence commis }\end{array}$ & C2 & Attitudes négatives & R2 & $\begin{array}{l}\text { Exposition à des } \\
\text { facteurs déstabilisants }\end{array}$ \\
\hline & à un jeune âge & C3 & $\begin{array}{l}\text { Symptômes actifs de } \\
\text { maladie mentale }\end{array}$ & R3 & Manque de \\
\hline H3 & $\begin{array}{l}\text { Instabilite des } \\
\text { relations intimes }\end{array}$ & & grave & & soutien personnel \\
\hline H4 & Problèmes d'emploi & & Impulsivité & R4 & $\begin{array}{l}\text { Inobservation des } \\
\text { mesures curatives }\end{array}$ \\
\hline H5 & $\begin{array}{l}\text { Problèmes de } \\
\text { toxicomanie }\end{array}$ & C5 & $\begin{array}{l}\text { Résistance au } \\
\text { traitement }\end{array}$ & R5 & Stress \\
\hline H6 & $\begin{array}{l}\text { Maladie mentale } \\
\text { grave }\end{array}$ & & & & \\
\hline H7 & Psychopathie & & & & \\
\hline $\mathrm{H} 8$ & $\begin{array}{l}\text { Inadaptation durant } \\
\text { l'enfance et } \\
\text { l'adolescence }\end{array}$ & & & & \\
\hline H9 & $\begin{array}{l}\text { Troubles de la } \\
\text { personnalité }\end{array}$ & & & & \\
\hline $\mathrm{H} 10$ & $\begin{array}{l}\text { Échec antérieur } \\
\text { de la surveillance }\end{array}$ & & & & \\
\hline
\end{tabular}

les faits, deux tendances apparaissent. La première aborde la prédiction du risque selon une approche actuarielle ; l'approche du Violence Risk Appraisal Guide (VRAG) (Quinsey et al., 1998) est précisément de ce type (tableau 1). L'objectif ici n'est pas de comprendre pourquoi tel ou tel aspect est lié au comportement violent ; il est uniquement de prédire le comportement violent d'un individu en s'appuyant essentiellement sur une probabilité statistique. La seconde tendance est représentée par l'instrument de Webster et al. (1997), soit la HCR-20 (tableau 2). Il n'est plus seulement question de l'évaluation du risque, mais également de la gestion du risque. Aux indices historiques (comportement violent antérieur, précocité des problèmes de comportement, trouble mental, indice de psycho- 
pathie, etc.) s'ajoutent maintenant des indices cliniques actuels et des indices liés au contexte futur dans lequel le patient est appelé à vivre. Les auteurs de la HCR-20 présentent l'instrument comme un aide-mémoire et non comme un instrument psychométrique. Cette tendance se situe à mi-chemin entre une approche quantitative et une approche qualitative. D'une part, chaque item est évalué sur la base d'un score de 0,1 ou 2 selon que l'individu rencontre les critères définis à l'item ; chaque souséchelle et l'échelle globale sont le résultat de la sommation des scores à chacun des items. D'autre part, le risque estimé ne se définit pas uniquement sur la base du résultat à l'échelle ; le poids relatif de divers items est également pris en compte dans l'estimation finale du risque. Ainsi, un individu qui présente un résultat élevé à l'indice de psychopathie (H7) sera considéré plus à risque de comportements violents qu'un individu qui obtient un résultat semblable à la HCR-20 mais pour lequel le résultat repose essentiellement sur l'instabilité relationnelle (H3), un trouble de la personnalité (H9), etc. En effet, sans que cela soit précisé de façon formelle dans le manuel, l'évaluation finale exige une part de jugement clinique. À la différence de l'approche actuarielle, les items ne sont pas statistiquement pondérés. Ainsi, la conclusion finale reposera sur le score certes, mais également sur un certain nombre de déductions faites à partir de l'expérience clinique.

Dans les faits, l'approche actuarielle et l'approche de l'évaluation et de la gestion du risque s'avèrent l'une et l'autre intéressantes. Plusieurs études montrent que ces instruments sont valides, spécialement les deux instruments auxquels il est fait référence, à savoir le VRAG et la HCR20 (Quinsey et al., 1998 ; Ross et al., 1998 ; Ross et al., 1999 ; Strand et al., 1999 ; Belfrage et al., 2000 ; Grann et al., 2000). Dès lors, nous assistons à un certain engouement pour ces instruments : la littérature scientifique y fait de plus en plus référence, diverses instances décisionnelles (juges, membres des commissions d'examen, notamment) identifient ces instruments et divers cliniciens cherchent à les utiliser, ce qui donne lieu à un accroissement des demandes de formation pour l'utilisation de ces instruments. Toutefois, l'intérêt croissant porté à ces instruments ne devrait pas faire oublier leurs limites.

\section{Mise en perspective critique}

Certes, le développement des instruments d'évaluation du risque a amélioré nettement la capacité de prédire les comportements violents, 
mais il appert que certaines limites peuvent être soulignées. À ce chapitre, les remarques se situeront à trois niveaux, soit aux niveaux méthodologique, épistémologique et éthique.

\section{Remarques au plan méthodologique}

Inévitablement, le premier point auquel un chercheur est porté à s'intéresser, c'est celui de la généralisation des résultats, laquelle dépend de la représentativité des échantillons à partir desquels les instruments ont été développés. À ce chapitre, Borum (1996) manifeste quelques réserves quant à la généralisation possible des résultats du VRAG ${ }^{1}$. Sa remarque s'appuie sur le fait que l'instrument a été développé auprès de sujets qui possédaient un passé de violence important, de sorte que, jusqu'à preuve du contraire, il reste à démontrer que les résultats sont les mêmes auprès d'une population générale de sujets atteints de troubles mentaux ou de troubles de la personnalité.

Par ailleurs, il apparaît plus préoccupant de constater que les auteurs appuient parfois la possibilité de généraliser leurs résultats sur la base de l'hétérogénéité de l'échantillon (Quinsey et al., 1998), même si la démonstration a été faite que l'instrument était aussi efficace dans le groupe des sujets qui sont passés à Oak Ridge pour une évaluation seulement, dans le groupe des sujets ayant été évalués puis incarcérés par la suite, comme dans le groupe des sujets qui ont été admis dans le programme de traitement. Il faut retenir que, dans ce dernier cas également, se trouvent un certain nombre de psychopathes, tels que définis à l'échelle de psychopathie de Hare (PCL-R) (Hare, 1991). Il appert que l'échantillon qui a servi à la validation du VRAG est hétérogène, comprenant à titre d'exemple $23 \%$ de schizophrènes, des psychopathes, des sujets présentant un trouble de la personnalité, etc. Par ailleurs, il est difficile d'avoir une idée précise de la composition de l'échantillon en ce qui a trait au diagnostic, puisque cette information n'est pas disponible de façon exhaustive dans les divers textes consultés. Dans le même sens, les auteurs de la HCR-20 ont souhaité développer un instrument qui puisse être utilisé dans divers milieux, y compris dans le milieu carcéral. Webster et al. (1997) précisent que l'utilisation de la HCR-20 est limitée aux milieux où se trouve une forte proportion de sujets présentant un passé de violence, et une «forte probabilité (strong suggestion) d'un

1. Borum réfere au Violence Prediction Scheme (Webster et al., 1995), la première appellation de ce qui est devenu le VRAG par la suite. 
trouble mental ou d'un trouble de la personnalité » (Webster et al., 1997 : 5). Toutefois, il est difficile de penser que les variables qui permettent de prédire le comportement violent à venir soient les mêmes chez les sujets psychotiques que chez les sujets présentant essentiellement un trouble de la personnalité. Nous n'avons ici qu'à penser au fonctionnement du psychopathe en comparaison de celui du sujet psychotique, joint au fait qu'il n'y a pas de réel recoupement entre l'un et l'autre diagnostics (Hodgins et al., 1998 ; Côté et al., données non publiées). Les analyses étant linéaires et basées sur les tendances centrales, le sous-groupe comprenant le plus de sujets influencera le poids des variables. Ainsi, il est intéressant de noter que le VRAG s'avère plus efficace dans le cas des sujets atteints d'un trouble de la personnalité comparativement aux sous-échelles clinique et risque de la HCR-20; ces deux dernières échelles s'avèrent plus efficaces dans le cas des sujets atteints d'un trouble mental grave (Grann et al., 2000).

Dans le même sens, ces instruments sont validés en considérant que les comportements violents se répartissent sur un continuum allant des voies de fait à l'homicide. Il est reconnu que les facteurs associés à certaines formes de violence ne sont pas les mêmes que pour une autre forme de violence. Il s'agit là d'un des postulats de base reconnu par Monahan et Steadman (1994) au moment d'élaborer les principes devant prévaloir dans l'évaluation du risque de comportements violents et, partant de là, orienter la recherche dans le cadre du projet MacArthur, un projet qui constitue un point de référence dans le domaine. Ainsi, nous avons pu démontrer que les variables associées à l'homicide se distinguent des variables associées à d'autres formes de comportement violent dans le cadre de l'étude portant sur la prévalence des troubles mentaux dans les pénitenciers du Québec (Côté et Hodgins, 1992). Il est démontré également que la psychopathie n'est pas d'abord et avant tout associée à l'homicide (Hare, 1981 ; Hare et Jutai, 1983 ; Hare et McPherson, 1984 ; Williamson et al., 1987 ; Pham et al., 1997 ; Pham, 1998), même si elle est fortement associée aux comportements violents. Les observations n'invalident pas pour autant les instruments actuariels en particulier, mais elles en marquent plutôt les limites, limites à mettre ici au chapitre de l'erreur de mesure.

Au-delà de la définition même du comportement violent, à savoir notamment si tous les comportements violents se placent sur un même continuum, il est vraisemblable que les facteurs associés auxdits comportements violents varient en fonction du contexte dans lequel s'exprime l'acte violent. Ainsi, les variables actuarielles s'avèrent supérieures aux 
variables dynamiques pour prédire les comportements violents dans la communauté ; toutefois, les variables dynamiques, notamment cliniques, sont supérieures aux variables statiques (actuarielles) pour prédire les comportements violents en établissement (Ross et al., 1998).

L'absence d'un cadre théorique préalable à la sélection des variables limite le raffinement de ces instruments. Hodgins (1997) leur reproche notamment de ne pas s'intéresser à l'étiologie. Ainsi, chez les psychotiques, une typologie basée sur la précocité des problèmes permet de distinguer deux groupes de patients ; ces derniers présentent une évolution distincte de la maladie, posent des problèmes distincts de prise en charge, connaissent des difficultés distinctes avec les substances psychoactives (Hodgins et al., 1998). Dans le même sens, ce ne sont pas tous les sujets d'un trouble mental grave qui présentent un risque de comportement violent. Nous avons pu démontrer qu'il existe une spécificité clinique chez ceux qui passent à l'acte et que nous retrouvons, par exemple, dans le système correctionnel ; cette spécificité s'exprime au niveau des diagnostics de base. Ainsi, parmi les psychotiques, nous retrouvons davantage de troubles délirants et de diagnostics du trouble psychotique non spécifié chez les sujets du milieu carcéral que chez les sujets rencontrés en milieu psychiatrique (Côté et al., 1997).

Toutefois, cette façon d'aborder le problème engage une réflexion épistémologique, du fait que l'analyse privilégie une approche taxinomique par rapport à une approche linéaire.

\section{Remarques au plan épistémologique}

L'approche actuarielle repose essentiellement sur des facteurs associés. L'association est établie sur la base de la régularité des observations. Nous pouvons affirmer que tel facteur permet de prédire le comportement violent ou la récidive du fait que cette dernière issue est régulièrement précédée de tel ou tel indice ; cet indice peut être observé de façon concomitante, ce qui n'empêche pas de conclure au fait que l'un et l'autre sont associés. Pour être en mesure d'augmenter la capacité de prédire, le chercheur considérera plus d'un indice à la fois. Ainsi, par le biais d'un contrôle statistique de variables (le schème est corrélationnel), notamment en recourant aux régressions logistiques ou aux régressions multiples, celui-ci pourra établir l'apport direct d'un indice, ce qui lui permettra par la suite de les cumuler pour augmenter son taux de prédiction. Aussi sophistiquées que puissent être les techniques d'analyses statistiques, la base demeure l'étude des contingences ou des régularités. 
Au plan du fonctionnement cognitif, cela revient à l'établissement de lois régissant les rapports d'associations ; il n'y a ici aucune préoccupation et aucune possibilité d'explication.

Le clinicien peut-il se satisfaire d'une telle approche ? S'insère ici le débat actuel entre les tenants de l'approche actuarielle et ceux qui veulent qu'en plus des variables statiques, soient prises en considération les variables cliniques et dynamiques. Quinsey et al. (1998) ne reconnaissent aucune pertinence au fait d'élargir la considération des facteurs intervenants au contexte ou à des indices cliniques. Par ailleurs, Webster et al. (1997) rapportent un ensemble d'études qui associent certains symptômes positifs aux comportements violents chez les psychotiques. Pour ces derniers auteurs, comme il ne s'agit plus essentiellement de " prévoir la dangerosité » mais bien d'évaluer le risque, la démarche exige "que l'évaluation couvre un champ plus vaste, qui tient compte des particularités propres à la situation de l'individu et à son milieu social » (p. 4). Il a été démontré que divers facteurs du contexte interviennent dans la détermination du passage à l'acte, tel un faible niveau socio-économique du milieu de résidence (Silver et al., 1999) ou la qualité du suivi (Rice et al., 1992 ; Hodgins et al., 1996). Si tel est le cas, la préoccupation du clinicien est de ne pas en rester à des variables essentiellement statiques, mais de prendre également en considération des variables dynamiques, c'est-à-dire des variables qui sont susceptibles de se transformer en fonction de l'intervention, de l'évolution du patient, de même que des facteurs de situation qui interviennent ou risquent d'intervenir dans un contexte donné. Les auteurs aspirent à évaluer non seulement le risque, mais également la " gestion du risque ».

Dès lors qu'il ne s'agit plus seulement de prédire le risque, mais d'évaluer et de gérer le risque de comportements violents, n'entre-t-on pas de plain-pied dans l'étude des processus?

Il est difficile, voire impossible, d'en rester à l'étude des régularités lorsque les variables dynamiques sont prises en compte. D'une part, les variables dynamiques ne connaissent pas la stabilité que présentent les variables statiques, fluctuant en fonction des interventions, d'une dynamique personnelle, d'événements extérieurs, etc. D'autre part, ces variables paraissent plus interdépendantes en comparaison des variables statiques. Ainsi, la symptomatologie influence vraisemblablement l'introspection et, partant de là, la participation au traitement. Naturellement, la participation sera éventuellement influencée par la disponibilité dudit traitement. En somme, n'y a-t-il pas là un début d'analyse causale ? 
Si nous en restons à l'approche linéaire, l'analyse causale exige un schème de recherche impossible à mettre en place eu égard au problème abordé ; pensons ici au schème expérimental. Un contrôle de variables a posteriori, inscrit dans un schème corrélationnel, exigerait une taille d'échantillon que nous ne pouvons imaginer tellement elle est importante. Même les analyses qui se développent dans le champ de l'évaluation du risque demeurent d'approche linéaire, faisant ici référence aux arbres de classification nouvellement présentés par l'équipe du projet MacArthur (Steadman et al., 2000); Bergman et Magnusson (1997) cherchent à rompre avec l'approche linéaire en proposant des analyses non pas centrées sur les variables mais centrées sur les personnes. L'argumentation théorique pour ce faire s'appuie sur la théorie générale des systèmes de Von Bertalanffy $(1967,1968)$. Ce dernier définit un système comme un « complexe d'éléments en interaction ». Selon cette conception, une variable ne peut être étudiée en elle-même. Son apport ne peut être saisi sans que soient prises en considération les relations que cette variable établit avec une ou plusieurs autres variables. Dans le cadre de l'analyse linéaire, les différences observées entre les individus ne sont pas des différences de nature mais d'intensité. Sous cet angle, l'approche d'une variable est quantitative et non qualitative. Dans le cadre d'une approche holistique — telle l'approche générale des systèmes —, le tableau d'ensemble repose sur un agencement ; c'est, en effet, l'organisation d'ensemble qui fait sens et non une variable en particulier. Le tableau observé n'est plus quantitativement distinct du tableau présenté par un individu ou un groupe d'individus, mais il est qualitativement distinct. Au plan méthodologique, nous passons ainsi de l'analyse linéaire à l'analyse typologique, de l'approche linéaire à l'approche taxinomique ${ }^{2}$.

En fait, la prise en considération de variables dynamiques pose la limite de la méthodologie de recherche actuellement privilégiée. En ce sens, il est intéressant de noter que toutes les études visant à appuyer la valeur de la

2. Meehl (1954), dans un texte de référence sur les approches clinique et statistique, reconnaît l'importance de l'analyse taxinomique; il la considère comme envisageable dans le cadre de l'approche actuarielle. Toutefois, la définition qu'il donne de l'approche actuarielle est large ; elle réfere en définitive à toute forme de validation statistique de la prédiction initiale. Pour lui, l'approche actuarielle se caractérise par son degré d'explicitation et de précision ; elle permet ainsi d'évaluer la valeur de la prédiction établie. Dans son texte, l'approche clinique se caractérise par le côté indéfini du jugement porté et par l'absence d'un souci de valider ce dernier quant au risque présenté. Ainsi définie, la HCR-20 rencontre ses critères de l'approche actuarielle. Même si le texte date, l'auteur en défend encore la pertinence, soutenant " la supériorité de la prédiction actuarielle sur la prédiction clinique ” (Meehl, 1986). 
HCR-20 utilisent les scores à l'échelle et aux sous-échelles, alors que les items ne sont pas pondérés et que les auteurs précisent spécifiquement qu'il ne s'agit pas d'un instrument psychométrique mais d'un aidemémoire; du reste, nous avons déjà souligné le fait que l'évaluation du risque établi à l'aide de la HCR-20 exige une part de jugement clinique du fait qu'il ne repose pas d'abord et avant tout sur un score. En somme, nous avons là une démonstration que l'analyse des processus pose des défis à la méthodologie quantitative. Qu'il suffise de préciser que l'étude des processus relève en définitive des rapports de nécessité, ce qui est « le propre des liaisons logiques ou mathématiques » (Piaget, $1970: 112$ ), dépassant ainsi la simple considération des lois basées sur les régularités. La compréhension d'un processus ne peut reposer sur une approche inductive ; elle exige que nous reconnaissions la possibilité de la déduction. Ainsi posée, l'approche redonne une place importante au clinicien en ceci que la compréhension des processus exige, ce faisant, la reconnaissance de la conscience en tant qu' " activité cognitive sui generis » (Piaget, 1970 : 115), ce que ne peut pallier quelque forme d'approche actuarielle. Le problème n'est pas propre à la psychologie ou à la psychiatrie ; la nécessité de reconnaître une part active à la personne, clinicien ou chercheur, est également reconnue en sociologie tel que le rapporte Clavette (1994) dans son texte intitulé Recherches qualitatives et recherches quantitatives : un même univers épistémologique. Endossant la pensée des sociologues Daniel Bertaux et Alain Testart, elle soutient que la part du sujet pensant ne peut être évacuée : "L'observation la plus élémentaire appelle une pensée organisatrice » (Clavette, $1994: 11$ ).

Il apparait également que le fait d'aborder le problème de l'évaluation du risque en s'intéressant aux processus réintroduit la préoccupation pour l'intervention. D'ailleurs, il n'est plus seulement question actuellement de l'évaluation du risque mais également de la gestion du risque. Seul l'accès aux processus fournit des pistes d'intervention puisqu'ils reposent sur une compréhension des rapports nécessaires et non probabilistes entre les diverses variables engagées. Qui plus est, renoncer à l'idée que la personne dont on s'occupe puisse changer, c'est en définitive renoncer à l'intervention. Il s'agit d'un aspect clinique qui engage une réflexion au plan de l'éthique.

\section{Remarques au plan éthique}

Quinsey et al. (1998) prétendent qu'il est non éthique de ne pas utiliser les instruments actuariels. L'argumentation repose sur le fait qu'il est 
moins éthique d'utiliser le jugement clinique pour évaluer le risque étant donné que celui-ci est trop aléatoire. Néanmoins, des problèmes se posent sur le plan éthique lorsque l'évaluation du risque de comportements violents repose essentiellement sur des variables statiques; par définition, les aspects auxquels réferent ces variables ne changent pas à travers le temps. L'apport de chacune de ces variables a été établi sur la base d'une approche nomothétique, c'est-à-dire d'une approche qui met l'accent sur les tendances centrales à l'intérieur d'un groupe. Si le clinicien doit tenir compte des changements intervenus chez son patient, éventuellement de l'état mental de ce dernier à un moment précis de son fonctionnement, celui-ci aura toujours à évaluer la pertinence du résultat obtenu à l'aide d'un instrument actuariel dans le cadre de l'évaluation de son patient. Selon Hart (1998), l'analyse actuarielle du risque a ses limites ; aux indices statiques issus de cette analyse, il importe de considérer des aspects "idiosyncrasiques ${ }^{3}$, en somme, des aspects individuels. La personne doit toujours demeurer au centre des préoccupations. Elle est vivante affectivement ; elle ne peut être abordée sur un plan essentiellement technique. Il importe de reconnaitre son potentiel de changement, ce qui exige un jugement clinique.

\section{Conclusion}

Pour les défenseurs de l'approche actuarielle, le recours au jugement du clinicien n'ajoute rien à la précision des résultats actuariels, si ce n'est même qu'il contribue à diminuer la valeur desdits instruments (Harris et Rice, 1997). Toutefois, rejeter l'apport des variables dynamiques ne correspond pas à ce qui peut être observé par ailleurs en ce qui a trait à l'importance de la symptomatologie et des aspects liés à la situation ou au contexte ; les possibilités offertes par le suivi paraissent démontrées et particulièrement convaincantes. Partant de là, les variables dynamiques sont prises en compte. L'introduction de ces variables ne joue pas un rôle complémentaire; elles questionnent toute l'approche de la recherche. Des réponses à ce nouveau questionnement exigent une réflexion épistémologique approfondie. Cette dernière amène à resituer l'individu au cœur de la démarche du risque, ce qui permet, du même coup, d'éviter un problème éthique.

3. Qualificatif utilisé par Hart lui-même. 


\section{Références}

Belfrage, H., Fransson, G. et Strand, S. 2000. «Prediction of Violence Using the HCR-20: A Prospective Study in Two Maximum-Security Correctional Institutions » Journal of Forensic Psychiatry 11 (1) : 167-175.

Bergman, L. R. et MAGnusson, D. 1997. «A Person-Oriented Approach in Research on Developmental Psychopathology" Development and Psychopathology 9 (2) : 291- 319.

Boer, D. P., Hart, S. D., Kropp, P. R. et Webster, C. D. 1997. The Sexual Violence Risk - 20 Guide (SVR-20), Burnaby, British Columbia, Canada: The Mental Health, Law and Policy Institute, Simon Fraser University.

Borum, R. 1996. "Improving the Clinical Practice of Violence Risk Assessment: Technology, Guidelines, and Training "American Psychologist $51: 945-956$.

ClavetTE, H. 1994. Recherches qualitatives et recherches quantitatives: un même univers épistémologique, Ste-Foy : Université Laval, Laboratoire de recherche, École de service social.

CotÉ, G. et Hodgins, S. 1992. «The Prevalence of Major Mental Disorders among Homicide Offenders » International Journal of Law and Psychiatry 15 : 89-99.

CÔtÉ, G., Lesage, A., Chawky, N. et Loyer, M. 1997. « Clinical Specificity of Prison Inmates with Severe Mental Disorders: A Case-Control Study » British Journal of Psychiatry 170 : 571-577.

Grann, M., Belfrage, H. et Tengström, A. 2000. « Actuarial Assessment of Risk for Violence: Predictive Validity of the VRAG and the Historical Part of the HCR-20 » Criminal Justice and Behavior 27 (1) : 97-114.

HANSON, K. et THORnTON, D. 2000. «Improving Risk Assessments for Sex Offenders: A Comparison of Three Actuarial Scales » Law and Human Behavior 24 (1) : 119-136.

HARE, R. D. 1991. The Hare Psychopatby Checklist: Revised, Toronto, Ontario : Multi-Health Systems, Inc.

HARE, R. D. 1981.«Psychopathy and Violence », Pp. 53-74 in Violence and the Violent Individual, sous la direction de J. R. Hays, T. K. Roberts et K. S. Soloways . Jamaica, NY : Spectrum.

Hare, R. D. et JutaI, J. W. 1983. « Criminal History of the Male Psychopath: Some Preliminary Data », Pp. 225-236 in Studies of Crime and Delinquency, sous la direction de K. T. Van Dusen et S. A. Mednick. Boston, MA : Kluwer Nijhoff Publishing.

Hare, R. D. et MCPherson, L. M. 1984. « Violent and Aggressive Behavior by Criminal Psychopaths » International Journal of Law and Psychiatry 7 : 35-50.

HARris, G. T. et RiCE, M. E. 1997. «Risk Appraisal and Management of Violent Behavior », Psychiatric Services 48 : 1168-1176.

HART, S. D. 1998. « The Role of Psychopathy in Assessing Risk for Violence: Conceptual and Methodological Issues » Legal and Criminological Psychology $3: 121-137$. 
Heilbrun, K., Philipson, J., Berman, L. et Warren, J. 1999. «Risk Communication: Clinicians' Reported Approaches and Perceived Values » Journal of the American Academy of Psychiatry and the Law 27 : 397-406.

HodGINS, S. 1997. "An Overview of Research on the Prediction of Dangerousness » Nordic Journal of Psychiatry 5 (Suppl. 39) : 33-38.

Hodgins, S., CôTÉ, G. et Toupin, J. 1998. « Major Mental Disorder and Crime: An Ethiological Hypothesis », Pp. 231-256 in Psychopatby: Theory, Research and Implications for Society, sous la direction de D. Cooke, A. Forth et R. D. Hare. Dordrecht, The Netherlands : Kluwer.

HODGINS, S., TOUPIN, J., FISET, S. et MOISAN, D. 1996. Soins communautaires pour patients souffrant de troubles mentaux graves et ayant une bistoire d'actes illégaux: Une comparaison de l'efficacité des soins spécialisés et non-spécialisés, Rapport de recherche présenté au Conseil Québécois de la Recherche sociale, Montréal, Université de Montréal, Département de psychologie.

Kropp, P. R., Hart, S. D., Webster, C. D. et EAves, D.1995. Spouse Assault Risk Assessment guide (SARA), Toronto : Multi-Health Systems Inc.

MCNIEL, D. E. et BINDER, R. L. 1994. «Screening for Risk of Inpatient Violence: Validation of an Actuarial Tool » Law and Human Behavior 18 : 579586.

MeEHL, P. E. 1954. Clinical versus Statistical Prediction: A Theoretical Analysis and a Review of the Evidence, Minneapolis : University of Minnesota Press.

MeEHL, P. E. 1986. « Causes and Effects of my Disturbing Little Book » Journal of Personality Assessment $50: 370-375$.

Monahan, J. 1981. Predicting Violent Bebavior: An Assessment of the Clinical Techniques, Beverly Hills (CA) : Sage.

Monahan, J. et Steadman, H. J. 1994. " Toward a Rejuvenation of Risk Assessment Research », Pp. 1-18 in Violence and Mental Disorder Developments in Risk Assessment, sous la direction de J. Monahan et H.J. Steadman. Chicago, IL : The University of Chicago Press.

PHAM, T. H. 1998. "Évaluation psychométrique du questionnaire de la psychopathie de Hare auprès d'une population carcérale belge » L'Encéphale XXIV : 435-441.

Pham, T. H., Remy, S., Dailliet, A. et Lienard, L. 1997. "Psychopathy and Prediction of Violent Behaviors: An Assessment in Security Hospital », Poster presented at The 5th International Congress on the Disorders of Personality, Vancouver, British Columbia, Canada.

PiAGET, J. 1970. Épistémologie des sciences de l'homme, Paris : Gallimard, Collection Idées.

Polvi, N. H. 1997. « Assessing Risk of Suicide in Correctional Settings », Pp. 278-301 in Impulsivity: Theory, Assessment, and Treatment sous la direction de C.D. Webster et M.A. Jackson. New York : Guilford.

Quinsey, V. L., Harris, G. T., Rice, M. E. et Cormier, C. A. 1998. Violent Offenders: Appraising and Managing Risk, Washington, DC: American Psychological Association. 
Rabinowitz, J. et GareliK-WyLer, R. 1999. «Accuracy and Confidence in Clinical Assessment of Psychiaric Inpatients Risk of Violence » International Journal of Law and Psychiatry 22 : 99-106.

Rice, M. E., Harris, G. T. et Cormier, C. A. 1992. «An Evaluation of a Maximum Security Therapeutic Community for Psychopaths and other Mentally Disordered Offenders » Law and Human Behavior 16 : 399-412.

Ross, D., Hart, S.D., Eaves, D. et Webster, C.D. 1999. « The Relationship between the HCR-20 and BC Review Board Decisions on the Release of Forensic Psychiatric Inpatients », Paper presented at the Conference on Risk Assessment and Risk Management: Implications for the Prevention of the Violence, Vancouver, British Columbia, Canada.

Ross, D. J., Hart, S. D. et Webster, C. D. 1998. Aggression in Psychiatric Patients: Using the HCR-20 to Assess Risk for Violence in Hospital and in the Community, Port Coquitlam, BC, Canada : Riverview Hospital Impulsivity Project.

Silver, E., Mulvey, E. P. et Monahan, J. 1999.«Assessing Violence Risk among Discharged Psychiatric Patients : Toward an Ecological Approach » Law and Human Behavior 23 : 237-255.

Steadman, H. et CocozzA, J. 1974. Careers of the Criminally Insane, Lexington, MA : Health.

Steadman, H., Silver, E., Monahan, J., Appelbaum, P. S., Clark Robbins, P., MulveY, E. P., Grisso, T., ROTH, L. H. et BANKS, S. 2000. « A Classification Tree Approach to the Development of Actuarial Violence Risk Assessment Tools » LAW AND HUMAN BEHAVIOR 24 : 83-100.

Strand, S., Belfrage, H., Fransson, G. et Levander, S. 1999. « Clinical and Risk Management Factors in Risk Prediction of Mentally Disordered Offenders - More Important than Historical Data? » Legal and Criminological Psychology 4 : 67-76.

ThORnBerry, T. P. et JACOBY, J. E. 1979. The Criminally Insane: A Community Follow-up of Mentally Ill Offenders, Chicago : The University of Chicago Press.

Von BertalanfFy, L. 1968. General System Theory: Foundations, Development, Applications, New York : George Braziller.

Von Bertalanffy, L. 1967. Robots, Men and Minds: Psychology in the Modern World, New York : George Braziller.

Webster, C. D., Eaves, D., Douglas, K. et Wintrup, A. 1995. The HCR-20 Scheme: The Assessment of Dangerousness and Risk (Version 1), Burnaby, BC, Canada: Simon Fraser University and Forensic Psychiatric Services Commission of British Columbia.

Webster, C.D., Douglas, K. S., Eaves, D. et Hart, S. D. 1997. « Assessing Risk of Violence to Others », Pp. 251-277 in Impulsivity: Theory, Assessment, and Treatment sous la direction de C. D. Webster et M.A. Jackson. New York : Guilford.

Williamson, S., Hare, R. D. et WONG, S. 1987. "Violence: Criminal Psychopaths and Their Victims» Canadian Journal of Bebavioural Science 19 : 454-462. 